medecine/sciences 1986; $2: 107$

\section{Localisation de la zone épileptogène}

Malgré de multiples recherches, il n'a pas été possible de localiser un foyer unique à l'origine des crises convulsives bilatérales. Des régions du cerveau, l'hippocampe ou l'amygdale notamment, ont fait l'objet d'explorations qui ont abouti à des conclusions contestées. C'est en examinant d'autres zones que Piredda et Gale à Washington (DC) pensent avoir résolu le problème grâce à une méthode stéréotaxique miniaturisée, assurant une précision inférieure à I mm. On sait que des crises peuvent être provoquées par des agents chimiques, avant tout par des antagonistes de l'acide gamma aminobutyrique (GABA) comme la bicuculline mais aussi des cholinergiques (carbachol), des acides aminés (acide glutamique, ainsi que son analogue structural, l'acide kainique, 50 fois plus actif que lui). A l'aide de canules guidées par stéréotaxie, ils ont pratiqué dans le cerveau des microinjections unilatérales de ces convulsivants à des doses de l'ordre de la picomole, et étudié les résultats cliniques et électroencéphalographiques.

Une seule zone, située dans le cortex profond prépiriforme, répond par des crises cloniques bilatérales. Les doses employées sont environ 20 fois plus faibles que celles qui provoquaient une réponse, habituellement unilatérale, dans les zones antérieurement suspectées.

De plus, la précision de la localisation est remarquable puisqu'il suffit de s'en écarter de I à $2 \mathrm{~mm}$ pour que les effets disparaissent. Enfin, l'application préalable d'un agoniste du GABA, le muscimol, empêche l'apparition des crises déclenchées par tous les agents actifs, alors que l'atropine ne prévient que celles qui sont dues au carbachol.

Les auteurs concluent que le cortex profond prépiriforme pourrait être l'origine d'excitations adressées aux structures limbiques et corticales sensibles. En outre, si ce foyer déclenche des crises sous l'influence d'agents différents, il pourrait représenter un dénominateur commun pour des modèles de crises considérées jusqu'alors comme dépendantes de circuits neuronaux distincts. Si ces résultats sont confirmés, il restera à établir les connexions fonctionnelles entre ce site et d'autres zones excitables notamment dans la région limbique, puis à déterminer si les données expérimentales obtenues chez le rat peuvent, s'appliquer à l'homme, grâce aux travaux parallèles obtenus par la méthode stéréotaxique.

J.-C. D.

Piredda S, Gale K. A crucial epileptogenic site in the deep prepiriform cortex. Nature 1985; 317 : 623-5.

\section{Angiogénine}

C'est dans une revue spécialisée de biochimie qu'en trois articles [I-3] Bert Vallee et coll. (Harvard) ont décrit l'isolement et le clonage d'une protéine humaine, l'angiogénine. Pourtant cette découverte semble d'une importance telle qu'elle a très rapidement fait l'objet d'éditoriaux dans Science et dans Nature $[4,5]$. Il s'agit en effet de la première protéine capable de stimuler la croissance des vaisseaux sanguins dont on ait identifié et la séquence et le gène.

Ce travail qui vient d'aboutir a commencé voici dix ans, lorsque la Monsanto $\mathrm{C}^{\mathrm{ie}}$ a offert une subvention de 23 millions de dollars, destinée aux recherches de Fudah Folkman sur le tumor angiogenesis factor; l'idée de départ était que les cancers solides devaient, pour se développer, posséder un facteur capable de leur assurer une vascularisation suffisante. Les efforts poursuivis pour isoler ce facteur à partir de cultures endothéliales de rat n'avançant que lentement, l'équipe de Vallee se sépara de celle de Folkman et choisit comme matériel les cellules de carcinome du colon humain en culture. De 2000 litres de milieu de culture, elle isola un milligramme d'une protéine pure, d'une masse moléculaire de 14000, formée de 123 acides aminés dont elle détermina la séquence. Il suffit de 3,5 picomoles, (une picomole $=10^{-12}$ mole), pour induire la formation de vaisseaux dans la cornée du lapin. Un oligonu- cléotide construit d'après un fragment de la séquence des acides aminés a permis l'obtention de l'ADN complémentaire issu d'une banque de foie humain, puis du gène. La séquence complète des nucléotides de l'ADNc et du gène a été analysée. Elle réservait deux surprises : le gène ne contient pas d'introns; d'autre part il présente, comme la protéine d'ailleurs, 35\% d'homologie avec la ribonucléase pancréatique; ce degré d'homologie implique une origine commune, bien qu'aucune des deux protéines ne possède les fonctions de l'autre. L'angiogénine a été isolée à partir de cellules tumorales, mais elle existe probablement, $\grave{a}$ des concentrations plus faibles, dans des tissus normaux et a déjà été mise en évidence dans le foie fatal. Elle n'est bien entendu pas le seul facteur de l'angiogenèse, mais elle suffit à la déclencher, et sa présence semble nécessaire pour la maintenir : en effet, les vaisseaux sanguins induits par un implant d'angiogénine dans la cornée du lapin régressent si on retire l'implant. Le clonage de langiogénine permettra d'en obtenir de grandes quantités et donc à la fois d'en préciser le rôle et probablement d'analyser la régulation de l'angiogenèse. Surtout, on voit se dessiner des espoirs thérapeutiques: il devrait être possible, en activant ou en inhibant la production d'angiogénine, de modifier la croissance des vaisseaux sanguins et d'agir sur celle des tumeurs en réduisant leur vascularisation. On ne peut encore prévoir les développements dans ce domaine, mais si l'angiogénine possède effectivement un avenir thérapeutique, les 23 millions de dollars de la Monsanto n'auront pas été une subvention à fonds perdus...

J.-C. D.

1. Fett JW, Strydom DY, Lobb RR, et al. Isolation and characterization of angiogenin, an angiogenic protein from human carcinoma cells. Biochemestry 1985; $24: 5480-6$.

2. Strydom DJ, Fett JW, Lobb RR, et al. Aminoacid sequence of human tumor derived angiogenin. Biochemistry 1985; 24 : 5486-94.

3. Kurachi K, Davie EW, Strydom DJ Sequence of the cDNA and gene for angiogenin, a human angiogenesis factor. Biochemistry 1985 ; 24: $5494-9$.

4. Marx JL. The 23-million dollar quest pays off. Science 1985; 230 : 161 .

5. Liotta $\mathrm{L}$. Isolation of a protein that stimulates blood vessel growth. Nature $1985 ; 318: 14$ 